\title{
A fast-response A-film-enhanced fringe field switching liquid crystal display
}

\author{
Haiwei Chen ${ }^{\mathrm{a}}$, Zhenyue Luo ${ }^{\mathrm{a}}$, Daming $\mathrm{Xu}^{\mathrm{a}}$, Fenglin Peng ${ }^{\mathrm{a}}$, Shin-Tson $\mathrm{Wu}^{\mathrm{a} *}$, Ming-Chun $\mathrm{Li}^{\mathrm{b}}$, Seok-Lyul Lee ${ }^{\mathrm{b}}$
} and Wen-Ching Tsai ${ }^{\mathrm{b}}$

${ }^{a}$ CREOL, The College of Optics and Photonics, University of Central Florida, Orlando, USA; ${ }^{b}$ AU Optronics Corp., Hsinchu, Taiwan

(Received 1 January 2015; accepted 30 January 2015)

\begin{abstract}
We propose a new A-film-enhanced fringe field switching (A-FFS) liquid crystal display whose required $d \Delta n$ value is only $\lambda / 4$, which is one-half of a conventional FFS. Fast response time can be achieved by either decreasing the cell gap $(d)$ or choosing a low birefringence $(\Delta n)$ and low-viscosity liquid crystal. The effect of $d \Delta n$ mismatch between A-film and FFS cell on contrast ratio is analysed. By choosing a dispersion-matched A-film, we can obtain a reasonably high contrast ratio in the entire visible region.
\end{abstract}

Keywords: liquid crystal display; fringe field switching; fast response time

\section{Introduction}

Fringe field switching (FFS) liquid crystal display (LCD) has been widely used in mobile displays and pads because of its outstanding features like high transmittance, wide view, weak colour shift, and pressure resistance for touch panels.[1-3] However, its response time is still relatively slow because the restoring elastic torque is mainly governed by the twisted elastic constant $K_{22}$. And in general $K_{22}$ is about $2 \mathrm{X}$ smaller than the other two elastic constants $K_{11}$ (splay) and $K_{33}$ (bend). For a $3-\mu \mathrm{m}$ cell gap the typical response time is about $10-20 \mathrm{~ms}$, depending on the viscosity of the LC employed.

To improve response time, several approaches have been proposed. The simplest method is to use an ultra-low viscosity LC material, $[4,5]$ because the response time is linearly proportional to the viscoelastic coefficient. Another option is to apply an erasing field to accelerate the LC relaxation process, such as triode structure,[6-8] dual fringe fields in a VA cell,[9] and crossed fringe fields.[10,11] However, these approaches are effective only for positive dielectric anisotropy $(\Delta \varepsilon)$ LCs. Moreover, these double-side striped electrodes require high precision registration; a small misalignment would significantly degrade the transmittance.[8] Last but not least, thin cell approach.[12] Because LC response time is proportional to the square of cell gap $\left(d^{2}\right)$, reducing cell gap is a straightforward way to shorten response time. Nevertheless, a minimum $d \Delta n$ value, where $\Delta n$ is the LC birefringence, should be maintained in order to achieve high transmittance.

In this paper, we propose a new FFS structure with an in-cell uniaxial A-film phase retarder, abbreviated as A-FFS mode. A key feature is that A-FFS can maintain high transmittance with a small $d \Delta n$ value. As a result, the response time can be improved without sacrificing the transmittance. With a dispersion-matched A-film, good dark state can be achieved in the whole visible wavelengths.

\section{Device structure}

Figure 1(a) shows the device configuration of conventional FFS structure. The LC directors in the voltageoff state are parallel to the optical axis of the input polariser. The incident linearly polarised light experiences no phase retardation after passing through the FFS cell, and is blocked by the crossed linear analyser, leading to a dark state. In the fully-on state, the FFS cell can be treated as a half-wave plate, through which the polarisation of the outgoing light is rotated by $90^{\circ}$, so that it passes through the analyser. Obviously, its $d \Delta n$ value has to be larger than $\lambda / 2$, otherwise the transmittance will decrease dramatically because of the insufficient phase retardation.

Figure 1(b) depicts the device configuration of our proposed A-FFS mode. A uniaxial A-film is sandwiched between the polariser and the FFS cell. The phase retardation of the A-film is the same as that of the FFS cell, i.e., $d_{A} \Delta n_{A}=d_{L C} \Delta n_{L C}$ and is equal to $\lambda /$ 4. The A-film together with the FFS cell functions as a new phase plate. For convenience, we call this A-film-enhanced FFS cell as A-FFS.

Similar to FFS, the bright state of A-FFS can be controlled by the applied voltage. At $V=0$, the optic axis of A-film is perpendicular to the LC directors (here we use a positive A-film) so that the total phase retardation is zero (subtractive), leading to a good dark state. As the voltage increases, the LC directors

*Corresponding author. Email: swu@ucf.edu 

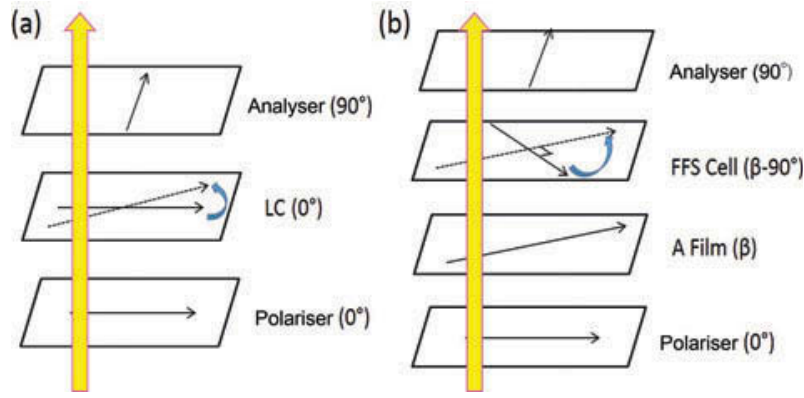

Figure 1. (Colour online) Device structure of (a) conventional FFS and (b) A-FFS LCDs.

are reoriented by the electric field and the phase retardation increases. When the additive phase retardation equals to $\lambda / 2$, a high transmittance state is obtained. Therefore, the new limit of A-FFS is $d_{A} \Delta n_{A}=d_{L C} \Delta n_{L C}=\lambda / 4$, since $d_{A} \Delta n_{A}-d_{L C} \Delta n_{L C}$ $=0$, and $d_{A} \Delta n_{A}+d_{L C} \Delta n_{L C}=\lambda / 2$. In comparison with FFS, the required $d \Delta n$ value for A-FFS is only one-half. This reduced $d \Delta n$ requirement can be utilised to improve response time in two-fold: (1) If $d$ is kept constant (same as FFS), then $\Delta n$ can be much smaller than the conventional one. Usually, the $\Delta n$ of liquid crystal used in FFS is 0.1 , but the $\Delta n$ of A-FFS can be as low as 0.05 . It gives us more freedom to choose low viscosity LCs for achieving faster response time. (2) If the $\Delta n$ is kept the same, then the cell gap of A-FFS can be reduced by $50 \%$, which in turn results in $4 \mathrm{X}$ faster response time. Of course, the final limiting cell gap is determined by the manufacturing technology.

\section{Simulation results}

The electro-optical properties of both conventional FFS and proposed A-FFS are calculated by a commercial LCD simulator DIMOS.2D and the extended Jones matrix.[13] The cell parameters are: electrode width $w=2 \mu \mathrm{m}$, electrode gap $g=3 \mu \mathrm{m}$, and rubbing angle $\varphi=10^{\circ}$. The LC material used here is a negative $\Delta \varepsilon$ LC material UCF-N1,[14] which has following physical properties: $K_{11}=12.0 \mathrm{pN}, K_{22}=6.2 \mathrm{pN}$, $K_{33}=13.5 \mathrm{pN}, \Delta n=0.119, \Delta \varepsilon=-3.8$, and $\gamma_{1}=122.7 \mathrm{mPas}$. The A-film used as in-cell phase retarder is Mylar with $\Delta n=0.049$ at $\lambda=550 \mathrm{~nm}$, and its thickness is chosen to have the same phase retardation as the $\mathrm{LC}$ cell. The tolerance analysis will be discussed later.

\subsection{Polarisation angle effect $(\beta)$}

FFS cell is not a perfect phase plate because of the nonuniform LC reorientation induced by the electric

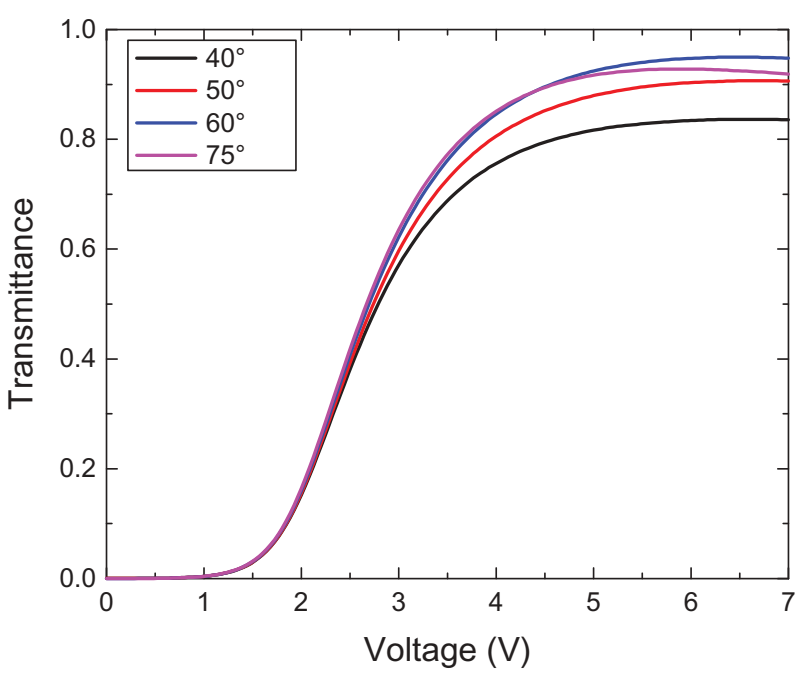

Figure 2. (Colour online) Simulated VT curves of A-FFS under different polarisation angles. LC: UCF-N1, $d \Delta n=275 \mathrm{~nm}$ and $\lambda=550 \mathrm{~nm}$.

field. In the on-state, n-FFS cell can be regarded as a double twisted nematic (TN) mode. Thus, we cannot determine the optimal angle between the polariser and A-film intuitively. Here, we define it as polarisation angle, labelled as $\beta$ in Figure 1(b). Figure 2 shows the simulated voltage-transmittance (VT) curves at different polarisation angles, and the highest transmittance is achieved at $\beta=60^{\circ}$. Afterwards, we do further investigation based on this optimal polarisation angle $\left(\beta=60^{\circ}\right)$.

\subsection{High transmittance}

For conventional FFS mode, even though the phase is large enough $(d \Delta n>\lambda / 2)$ the transmittance is still quite sensitive to the $d \Delta n$ value as shown in Figure 3. However, this is not the case for our new A-FFS. Because the phase is not determined by the FFS cell alone, it is the combinational effect of A-film and LC cell, in which A-film supplies an additional phase retardation to boost transmittance.

In our new A-FFS, we can still get high transmittance even though the phase retardation of LC cell decreases, e.g. cell gap decreases. As shown in Figure 3, A-FFS has the same transmittance as conventional FFS for $d \Delta n \geq 330 \mathrm{~nm}$. However as $d \Delta n$ decreases, our A-FFS keeps over $90 \%$ transmittance, but the transmittance of conventional FFS decreases rapidly. Therefore, A-FFS mode has high transmittance, which is insensitive to $d \Delta n$ variation.

\subsection{Response time}

For FFS, its optimised $d \Delta n$ value is about $0.65 \lambda$ (or $d \Delta n \approx 360 \mathrm{~nm}$ ) in order to keep the operation voltage 


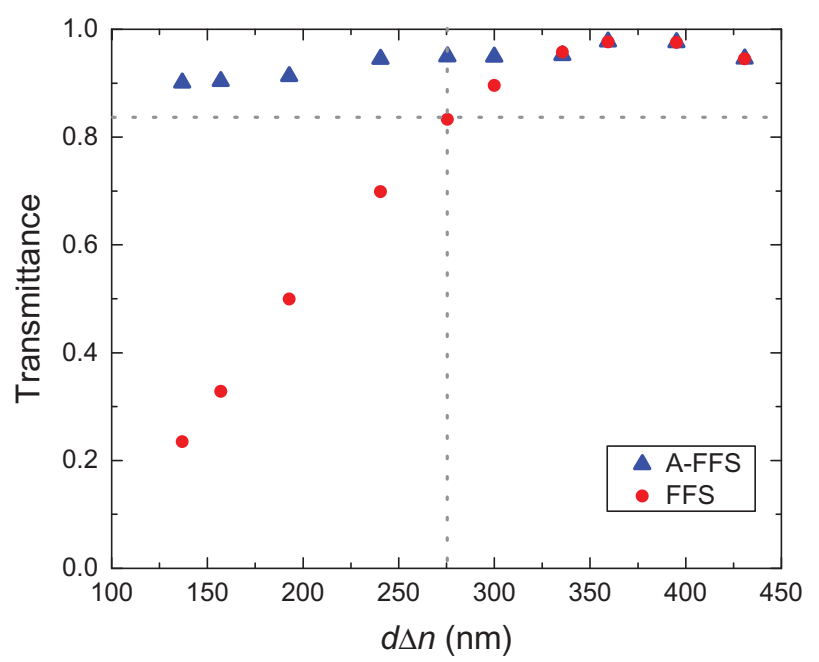

Figure 3. (Colour online) Simulated transmittance of A-FFS at $\lambda=550 \mathrm{~nm}$ with different $d \Delta \mathrm{n}$ values. The intersection of two dotted lines corresponds to $d \Delta n=\lambda / 2$.

below $6 \mathrm{~V}$, although its theoretical $d \Delta n$ limit is only $\lambda /$ 2.[13] Similarly for A-FFS, we need to increase $d \Delta n$ to about $0.5 \lambda$ (or $275 \mathrm{~nm}$ ) in order to keep a low operation voltage as depicted in Figure 2, although its theoretical $d \Delta n$ limit is only $\lambda / 4$. The smaller $d \Delta n$ requirement of A-FFS allows us to use a lower birefringence LC mixture (which usually exhibits a lower viscosity) or thinner cell gap. As cell gap decreases, response time decreases in proportion to $d^{2}$.[12]

\subsection{Cell gap tolerance}

A big challenge for A-FFS is that $d_{A} \Delta n_{A}=d_{L C} \Delta n_{L C}$ should be satisfied in order to obtain good dark state. What's more, the cell gap control error and temperature variation have to be taken into consideration. To evaluate the $d \Delta n$ tolerance, we calculate the contrast ratio of different cell gaps while keeping the A-film thickness unchanged. Besides, we try to optimise the dark state by tuning the polarisation angle $(\beta)$, and the results are listed in Table 1. From Table 1, the least tolerance occurs at $\beta=45^{\circ}$, and the tolerance increases with increasing $\beta$. At $\beta=80^{\circ}$, the tolerance range is $\pm 4.5 \%$ with $\mathrm{CR}>2000: 1$, and $\pm 8.6 \%$ with

Table 1. Tolerance of $d \Delta n$ under different criteria with different $\beta$ angles for $d_{A} \Delta n_{A}=d_{L C} \Delta n_{L C}=\lambda / 2$. LC: UCF-N1 with $d \sim 2.32 \mu \mathrm{m}, \lambda=550 \mathrm{~nm}$.

\begin{tabular}{ccccc}
\hline Contrast ratio & $>2000: 1(\%)$ & $>1000: 1(\%)$ & $>400: 1(\%)$ \\
\hline$d \Delta n$ & $\beta=45^{\circ}$ & \pm 0.85 & \pm 1.7 & \pm 3.25 \\
Tolerance & $\beta=60^{\circ}$ & \pm 1.3 & \pm 2.15 & \pm 3.45 \\
& $\beta=75^{\circ}$ & \pm 2.6 & \pm 3.85 & \pm 6.05 \\
& $\beta=80^{\circ}$ & \pm 4.5 & \pm 5.6 & \pm 8.6 \\
\hline
\end{tabular}

CR > 400:1, which is acceptable by the advanced fabrication technology. The above $d \Delta n$ tolerance analysis includes both cell gap and $\Delta n$ variations. The latter is caused by the temperature change; it can be mitigated by two methods: (1) using a high clearing point LC mixture and (2) choosing an A-film with similar temperature dependency as the employed LC material.

\subsection{Birefringence dispersion}

It is fairly easy to design an A-film to satisfy $d_{A} \Delta n_{A}=d_{L C} \Delta n_{L C}$ at a given wavelength, say $\lambda=550 \mathrm{~nm}$. However, the dispersion of both A-film and LC material could be mismatched at other wavelengths. For a full-colour display, the dark state of the proposed normally black A-FFS will be degraded if there exists a mismatched birefringence dispersion. To solve this problem, we have to choose the A-film and LC material carefully so that their dispersion remains the same in the whole visible region. Fortunately, this problem has been addressed two decades ago when dealing with wide viewing angle.[15]

The wavelength-dependent birefringence of liquid crystal could be described by [16]:

$$
\Delta n(\lambda)=G \frac{\lambda^{2} \lambda^{* 2}}{\lambda^{2}-\lambda^{* 2}}
$$

where $G$ is a proportionality constant and $\lambda^{*}$ is the mean electronic transition wavelength. For Mylar and UCF-N1, $\lambda^{*}$ is $224 \mathrm{~nm}$ [17] and $219 \mathrm{~nm},[18]$ respectively. Figure 4 depicts the wavelength dependent $\Delta n$ of these two materials. The $\Delta n$ shown in Figure 4 is normalised to the value at $\lambda=550 \mathrm{~nm}$ to

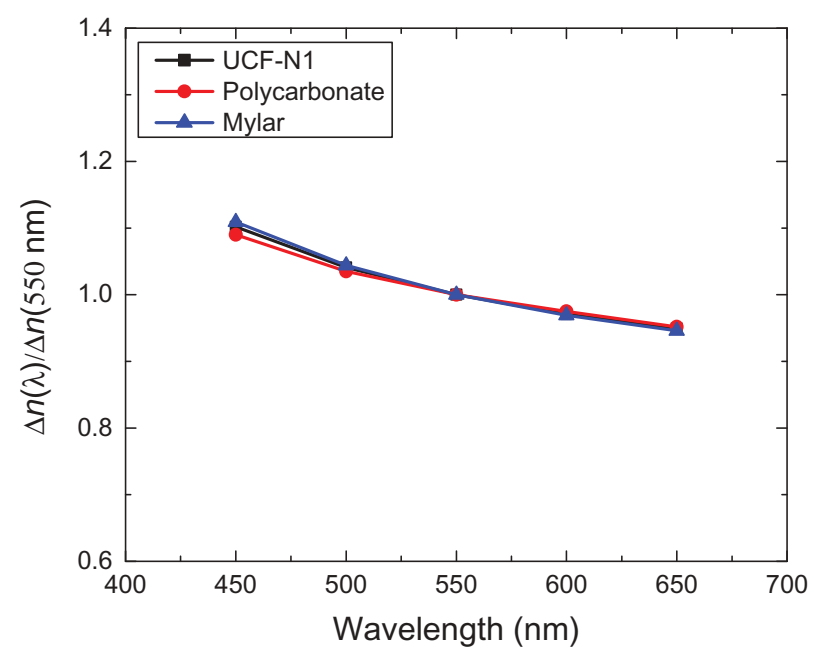

Figure 4. (Colour online) Wavelength dependent $\Delta n$ of UCF-N1, Polycarbonate and Mylar. 
avoid the scale difference. Excellent match is achieved in the visible region. Thus with Mylar as the A-film material, the contrast ratio for red $(650 \mathrm{~nm})$, green $(550 \mathrm{~nm})$ and blue $(450 \mathrm{~nm})$ is found to be 1784:1, 2914:1 and 1472:1, respectively. Besides Mylar, from Figure 4, Polycarbonate also matches quite well with UCF-N1, which means we can have more than one choice to get matched dispersion.[15] As the LC material changes, we need to find another matched A-film.

\subsection{The position of A-film}

All the results above are obtained with A-film sandwiched between the polariser and the LC cell, which means the input linearly polarised light passing through A-film first. Next, we investigate the order effect by exchanging the positions of A-film and LC cell, as illustrated in Figure 5(a) and (b). Theoretically speaking, the results should be the same whether the light comes from the polariser side (upward) or from the analyser side (downward). Figure 5(c) shows this effect, from which we can see the transmittance for both cases is the same except for a shifted phase $\left(\sim 90^{\circ}\right)$.

\subsection{Positive A-FFS}

The proposed A-FFS mode not only offers outstanding performance with negative $\Delta \varepsilon$ LC materials, but it also works well with positive LCs. Figure 6(a) and (b) shows the simulated transmittance and polarisation effect using a positive $\Delta \varepsilon$ LC material (MLC-6686,

(a)
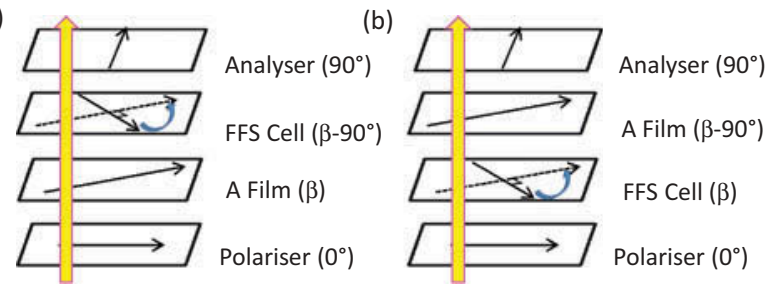

(c)

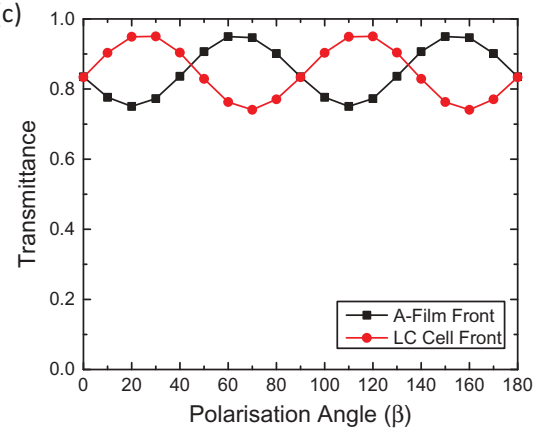

Figure 5. (Colour online) Device structures for (a) A-film in the front, (b) LC cell in the front, and (c) simulated transmittance at different polarisation angles.
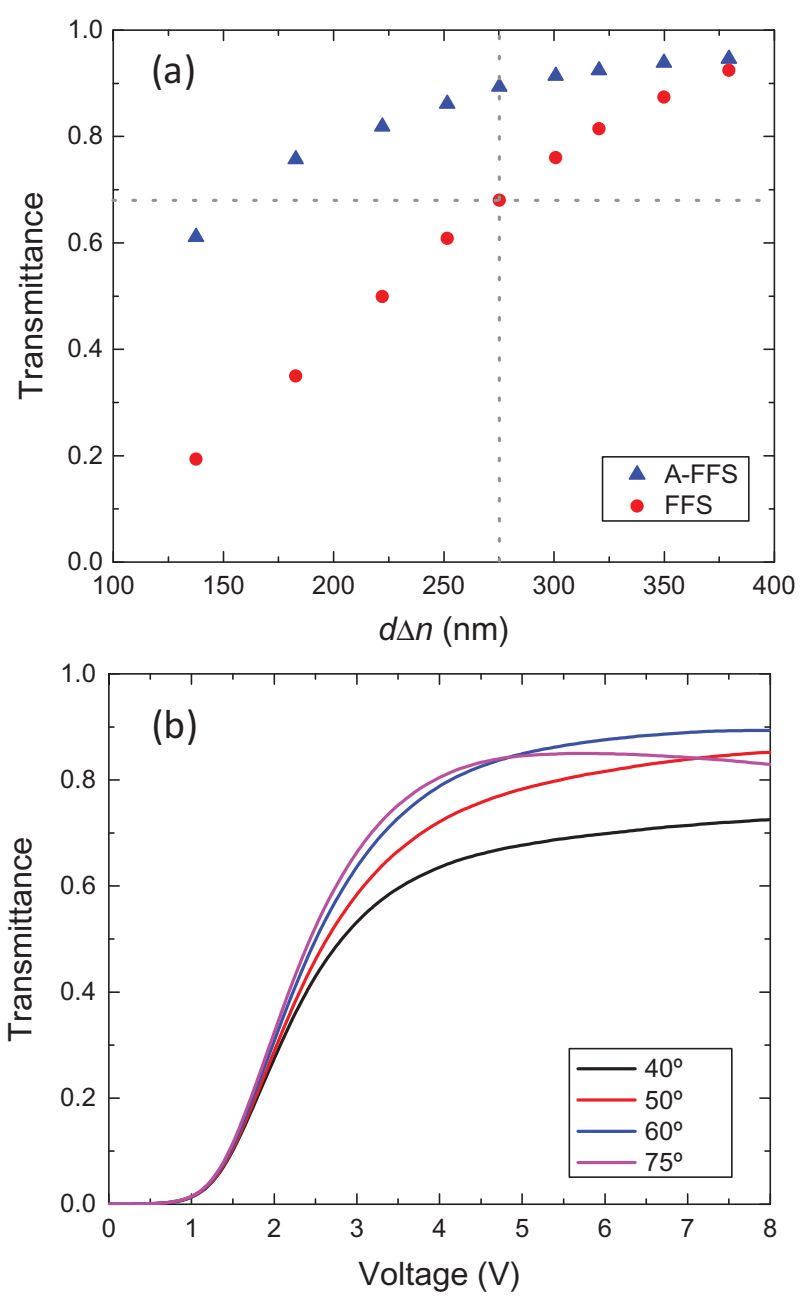

Figure 6. (Colour online) Simulated results for A-FFS using a positive $\Delta \varepsilon$ LC (MLC-6686) at $\lambda=550 \mathrm{~nm}$ : (a) transmittance with different cell gaps and (b) VT curves under different polarisation angles.

$\Delta n \sim 0.0983$ at $\lambda=550 \mathrm{~nm}$ ), respectively. Same as the negative A-FFS mode, the optimised polarisation angle for the positive A-FFS is also $60^{\circ}$. Nevertheless, the transmittance does not stay above $90 \%$ as the negative A-FFS shown in Figure 3. For a positive $\Delta \varepsilon \mathrm{LC}$, its directors tend to follow the electric field. In the middle of electrodes and gaps, the electric fields are largely in vertical direction. These vertically reoriented LCs do not contribute to transmittance. As a result, dead zones are formed and transmittance decreased. In spite of this shortcoming, A-FFS still exhibits a much higher transmittance than the corresponding FFS mode as Figure 6(a) depicts.

\section{Discussion}

The theoretical $d \Delta n$ limit for conventional FFS is $\lambda / 2$. However, the LC directors near the substrates are 
subject to strong surface anchoring so that they do not contribute to the phase retardation. In practical applications, we have to increase the $d \Delta n$ value to, say $0.65 \lambda$, obtain high transmittance and low operation voltage.[14] Similarly for A-FFS, its $d \Delta n$ value should be larger than the theoretical limit, which is $\lambda / 4$. During the above calculations, we set $d \Delta n=\lambda / 2$. Obviously, this value can be further decreased to enable a thinner cell gap for faster response time, but the major trade-off is increased voltage. To lower operation voltage, several approaches can be used, e.g. reducing the passivation layer thickness, decreasing the rubbing angle and increasing the dielectric anisotropy of the LC employed.[19]

For smart phones and pads operated by battery, low voltage helps to reduce power consumption and lengthen the battery's life. But for large-screen TVs or computer screens, a higher operation voltage $(7.5 \mathrm{~V})$ can be tolerated because the major power consumption comes from the backlight.[20] Especially for TVs, fast response time plays a key role to suppress motion picture image blurs. Under such condition, the advantages of A-FFS clearly manifest because it allows a thinner cell gap to be used for achieving fast response time. To illustrate this feature, we compare the VT curves of A-FFS and FFS at $d \Delta n=240 \mathrm{~nm}$, as depicted in Figure 7. From Figure 7, A-FFS reaches $95 \%$ transmittance at $7.5 \mathrm{~V}$, but the peak transmittance of FFS saturates at $70 \%$ although its voltage is only $6 \mathrm{~V}$.

For FFS mode, especially n-FFS $(\Delta \varepsilon<0)$, the electric-field-induced LC director reorientation takes place primarily in the horizontal direction, which results in very wide viewing angle. Since the working mechanism of A-FFS is the same as conventional FFS (in-plane molecular reorientation), the wide view property should be persevered.[21] Shown in Figure 8, A-FFS has a slightly degraded viewing angle, but the contrast ratio remains over 100:1 in

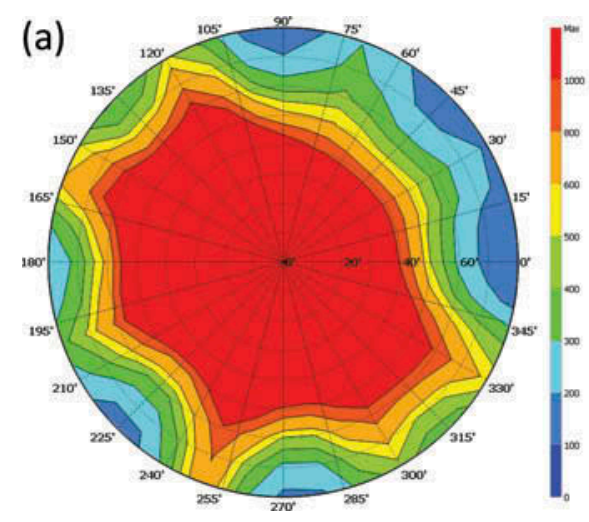

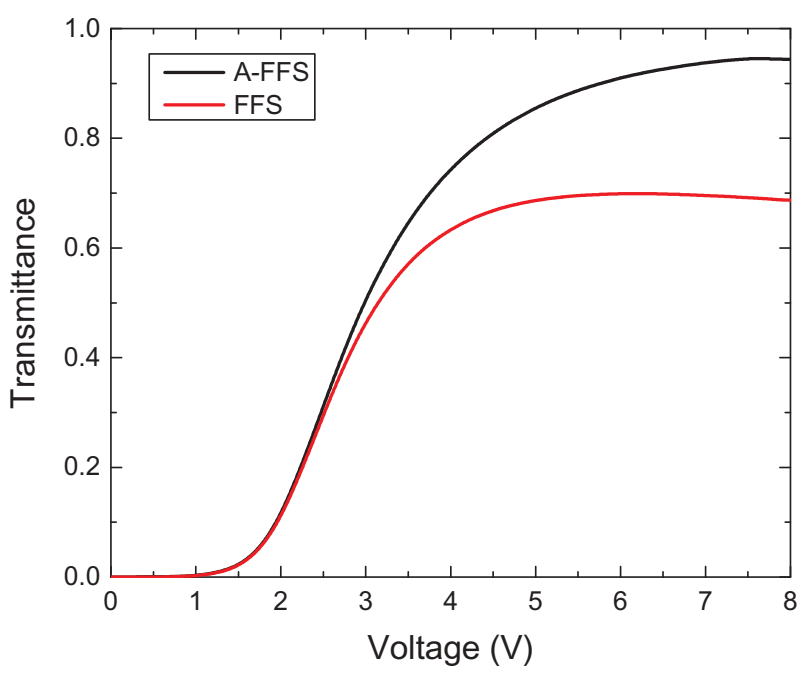

Figure 7. Simulated VT curves for A-FFS and FFS using $\mathrm{UCF}-\mathrm{N} 1$ at $\lambda=550 \mathrm{~nm}$ with $d \Delta n=240 \mathrm{~nm}$.

the entire $\pm 80^{\circ}$ viewing zone. What's more, with the emerging quantum dot (QD) enhanced backlight, the colour performance (e.g. colour shift and colour gamut) will be greatly improved. And superior image quality at outdoor environment and high optical efficiency could also be achieved.[22-24]

\section{Conclusion}

We proposed a new A-film-enhanced FFS cell which requires a much smaller $d \Delta n$ value than conventional FFS. It works by the combination of an A-film and a thin FFS cell: subtraction for dark state and addition for bright state. The new $d \Delta n$ limit for A-FFS mode is now $0.25 \lambda$, which makes two important impacts: (1) it offers more freedom to choose low-viscosity LC material if the cell gap is held constant and (2) it enables a thinner cell gap to be used if the LC birefringence is

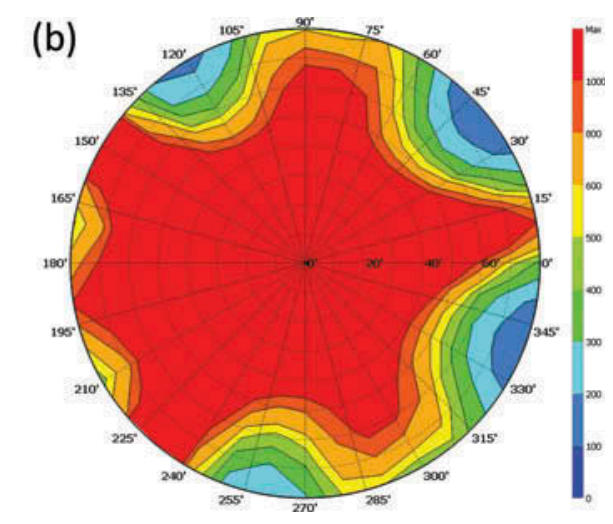

Figure 8. Simulated isocontrast contours for (a) A-FFS and (b) conventional FFS with a positive A-film and a negative C-film under $\lambda=550 \mathrm{~nm}$. 
kept the same. In addition, the A-FFS mode has some attractive features, such as intrinsically high transmittance, insensitive to cell gap and fast response time. The effect of cell gap and birefringence variations on dark state is a concern, but our simulation shows that a reasonably large tolerance in $d \Delta n$ is achieved. Also, by choosing a dispersion-matched A-film, good dark state is obtained in the entire visible range. Similar to FFS, the proposed A-FFS mode works for negative and positive $\Delta \varepsilon$ LC materials.

\section{Acknowledgement}

The authors are indebted to Dr Q. Hong for helpful discussion.

\section{Disclosure statement}

No potential conflict of interest was reported by the authors.

\section{Funding}

This work was supported by AU Optronics, Taiwan.

\section{References}

[1] Lee SH, Lee SL, Kim HY. Electro-optic characteristics and switching principle of a nematic liquid crystal cell controlled by fringe-field switching. Appl Phys Lett. 1998;73:2881-2883. doi:10.1063/1.122617.

[2] Lee SH, Kim HY, Lee SM, Hong SH, Kim JM, Koh JW, Lee JY, Park HS. Ultra-FFS TFT-LCD with super image quality, fast response time, and strong pressure-resistant characteristics. J Soc Inf Disp. 2002;10:117-122. doi:10.1889/1.1827852.

[3] Yun HJ, Jo MH, Jang IW, Lee SH, Ahn SH, Hur HJ. Achieving high light efficiency and fast response time in fringe field switching mode using a liquid crystal with negative dielectric anisotropy. Liq Cryst. 2012;39:1141-1148. doi:10.1080/02678292.2012.700078.

[4] Chen H, Peng F, Luo Z, Xu D, Wu S-T, Li M-C, Lee S-L, Tsai W-C. High performance liquid crystal displays with a low dielectric constant material. Opt Mater Exp. 2014;4:2262-2273. doi:10.1364/OME.4.002262.

[5] Zhan H-M, Xu Z, Wang Y-C, Lin L-F, Chen M, Kim W, Wang D, Shao X-B, Lee S-K. Fast response fringefield switching mode liquid crystal development for shutter glass 3D. J Soc Inf Disp. 2013;21:137-141. doi:10.1002/jsid.154.

[6] Channin DJ. Triode optical gate: a new liquid crystal electro-optic device. Appl Phys Lett. 1975;26:603. doi:10.1063/1.88018.

[7] Xiang CY, Guo JX, Sun XW, Yin XJ, Qi GJ. A fast response, three-electrode liquid crystal device. Jpn J Appl Phys. 2003;42:L763-765. doi:10.1143/JJAP.42.L763.

[8] Xu D, Rao L, Tu C-D, Wu S-T. Nematic liquid crystal display with submillisecond grayscale response time. J Disp Technol. 2013;9:67-70. doi:10.1109/JDT.2012.2232902.

[9] Jiao MZ, Ge ZB, Wu S-T, Choi W-K. Submillisecond response nematic liquid crystal modulators using dual fringe field switching in a vertically aligned cell. Appl Phys Lett. 2008;92:111101. doi:10.1063/1.2896650.

[10] Li Y, Ge ZB, Lu RB, Jiao MZ, Wu S-T. Fast-response liquid-crystal displays using crossed fringe fields. J Soc Inf Disp. 2008;16:1069-1074. doi:10.1889/JSID16.10.1069.

[11] Xiang CY, Sun XW, Yin XJ. Fast response wide viewing angle liquid crystal cell with double-side fringe-field switching. Appl Phys Lett. 2003;83:5154. doi:10.1063/ 1.1635982

[12] Ge Z, Wu S-T, Kim SS, Park JW, Lee SH. Thin cell fringe-field-switching liquid crystal display with a chiral dopant. Appl Phys Lett. 2008;92:181109. doi:10.1063/1.2918838.

[13] Lien A. Extended Jones matrix representation for the twisted nematic liquid-crystal display at oblique-incidence. Appl Phys Lett. 1990;57:2767-2769. doi:10.1063/1.103781.

[14] Chen Y, Luo Z, Peng F, Wu S-T. Fringe-field switching with a negative dielectric anisotropy liquid crystal. J Disp Technol. 2013;9:74-77. doi:10.1109/JDT.2013.2242844.

[15] Wu S-T. Phase-matched compensation films for liquid crystal displays. Mater Chem Phys. 1995;42:163-168. doi:10.1016/0254-0584(05)01571-5.

[16] Wu S-T. Birefringence dispersions of liquid crystals. Phys Rev A. 1986;33:1270-1274. doi:10.1103/ PhysRevA.33.1270.

[17] Wu S-T, Lackner AM. Mylar-film-compensated $\pi$ and parallel-aligned liquid crystal cells for direct-view and projection displays. Appl Phys Lett. 1994;64:2047. doi:10.1063/1.111731.

[18] Chen Y, Peng F, Yamaguchi T, Song X, Wu S-T. High performance negative dielectric anisotropy liquid crystals for display applications. Crystals. 2013;3:483-503. doi:10.3390/cryst3030483.

[19] Ryu JW, Lee JY, Kim HY, Park JW, Lee G-D, Lee $\mathrm{SH}$. Effect of magnitude of dielectric anisotropy of a liquid crystal on light efficiency in the fringe-field switching nematic liquid crystal cell. Liq Cryst. 2008;35:407-411. doi:10.1080/02678290801919659.

[20] Zhan H, Xu Z, Wang Y, Wang Y, Chen M, Kim W, Wang D, Shao X, Lee S, Zhao S. Low-voltage, high transmittance fringe-field switching mode liquid crystal for monitor display. Liq Cryst. 2014;41:755-760. doi:10.1080/02678292.2014.889234.

[21] Kang W-S, Moon J-W, Lee G-D, Lee S-H, Lee J-H, Kim B-K, Choi H-C. Retardation free in-plane switching liquid crystal display with high speed and wideview angle. J Opt Soc Korea. 2011;15:161-167. doi:10.3807/JOSK.2011.15.2.161.

[22] Luo Z, Chen Y, Wu S-T. Wide color gamut LCD with a quantum dot backlight. Opt Exp. 2013;21:2626926284. doi:10.1364/OE.21.026269.

[23] Jang E, Jun S, Jang H, Lim J, Kim B, Kim Y. Whitelight-emitting diodes with quantum dot color converters for display backlights. Adv Mater. 2010;22:30763080. doi:10.1002/adma.201000525.

[24] Luo Z, Xu S, Gao Y, Lee Y-H, Liu Y, Wu S-T. Quantum dots enhanced liquid displays. J Disp Technol. 2014;10:987-990. doi:10.1109/JDT.2014.2360627. 